\title{
La enfermedad diarreica aguda: un reto para la salud pública en Colombia
}

\author{
Diana Carolina Cáceres, ${ }^{1}$ Eduardo Estrada, ${ }^{2}$ Rodrigo DeAntonio ${ }^{1}$ \\ y Dioselina Peláez ${ }^{1}$
}

Forma de citar Cáceres DC, Estrada E, DeAntonio R, Peláez D. La enfermedad diarreica aguda: un reto para la salud pública en Colombia. Rev Panam Salud Publica. 2005;17(1):6-14.

RESUMEN Objetivos. Establecer la relación entre la infección por rotavirus y la deshidratación por diarrea en niños colombianos menores de 5 años y determinar los factores de riesgo de la diarrea con deshidratación.

Métodos. Se realizó un estudio de casos y testigos en un hospital urbano de Bogotá, Colombia, entre abril de 2000 y febrero de 2001. Se seleccionaron 290 niños menores de 5 años de edad de uno u otro sexo; de ellos 145 estaban hospitalizados por enfermedad diarreica aguda (EDA) con deshidratación (casos) y 145 tenían diagnóstico de EDA sin signos de deshidratación (testigos). A todos los niños se les realizó un examen físico completo. Las madres respondieron un cuestionario con variables demográficas; socioeconómicas; de conocimientos, actitudes y hábitos higiénicos; y de acceso a los servicios de salud. Se determinó la presencia de rotavirus en muestras de heces fecales tomadas durante las primeras 48 horas del ingreso al estudio.

Resultados. Se encontró una asociación estadística entre la diarrea con deshidratación y la presencia de rotavirus en las muestras de heces (razón de posibilidades $[R P]=3,46$; intervalo de confianza de 95\% [IC95\%]: 1,71 a 7,00), el peso al nacer < $2600 \mathrm{~g}(R P=7,79 ;$ IC95\%: 3,47 a 18,01) y el tiempo de lactancia materna inferior a 3 meses ( $R P=3,17 ;$ IC95\%: 1,66 a 6,13). El riesgo de presentar deshidratación se asoció con las condiciones socioeconómicas desfavorables, los hábitos higiénicos inadecuados de la familia del niño y una menor escolaridad materna. Conclusiones. La calidad inadecuada de las acciones de promoción y prevención en una población con fácil acceso a los servicios de salud favoreció la aparición de casos de diarrea con deshidratación. La infección por rotavirus desempeña un papel importante en la gravedad de la EDA en niños colombianos.

Palabras clave Diarrea infantil, deshidratación, factores de riesgo, rotavirus, Colombia.

A pesar del desarrollo y difusión de la rehidratación oral, la enfermedad

\footnotetext{
1 Instituto Nacional de Salud, Bogotá, Colombia. La correspondencia debe ser dirigida a Diana Carolina Cáceres, Instituto Nacional de Salud, Dirección General, Avenida El Dorado No. 51-60, Bogotá, Colombia. Correos electrónicos: dcaceres@ ins.gov.co; dianacaceres123@hotmail.com

2 Clínica Infantil Colsubsidio, Bogotá, Colombia.
}

diarreica aguda (EDA) continúa siendo una causa importante de morbilidad y mortalidad en niños menores de 5 años. Se calcula que en los países en desarrollo, los niños presentan entre 3 y 10 episodios de diarrea anuales durante los primeros 5 años de vida, con una duración promedio de estos episodios de 4 a 6 días. En 1992 se informaron aproximadamente 3,3 millones de muertes relacionadas con la diarrea en el mundo y entre 1995 y 2000 , esta enfermedad ocasionó $11,8 \%$ de las muertes en menores de 5 años (1-3).

De acuerdo con los datos de morbilidad y mortalidad correspondientes al período de 1989 a 1991, el Ministerio de Salud de Colombia calculó que las enfermedades transmisibles y perina- 
tales fueron responsables de $21 \%$ de los años de vida saludables perdidos por discapacidad en ese trienio (4). De igual forma, entre 1989 y 1997, la diarrea fue la segunda causa de hospitalización en niños menores de 1 año (entre 9 y $15 \%$ de los ingresos en este grupo de edad) y la segunda causa de hospitalización en niños de 1 a 4 años (entre 12 y 15\% de los niños hospitalizados de esa edad) (5).

La EDA continúa siendo un problema importante de salud pública por ser una de las principales causas de muerte en menores de 5 años (6), por el elevado número de casos que se presentan anualmente y por los gastos que genera el tratamiento médico general o específico de los enfermos.

No solo la deshidratación hace que aumenten los costos de la atención de los enfermos con diarrea. La intolerancia a la lactosa, que se desarrolla como consecuencia de la infección, puede afectar a un considerable número de casos (entre $7,5 \%$ y $77 \%$ ) y provocar que se prolongue el cuadro diarreico, que aumente la gravedad de la enfermedad y que se sobrecarguen aun más los servicios de salud (7). La desnutrición, otra complicación favorecida por las malas prácticas alimentarias de las madres o de los cuidadores de los menores, favorece la sobreinfección, aumenta la morbilidad y la mortalidad y eleva los costos de la atención sanitaria (7).

Entre los factores protectores de la EDA se encuentran la lactancia materna durante los primeros 4 meses de vida (8-10), los hábitos higiénicos adecuados - lavarse las manos y otras medidas de higiene personal- y la conservación adecuada de los alimentos. Algunos estudios han demostrado que con la aplicación de estas medidas se ha logrado reducir la mortalidad por EDA en $55 \%$ y que el saneamiento ambiental puede tener más impacto en la reducción de la prevalencia de la diarrea que otras medidas, como las dirigidas a mejorar el suministro de agua. Igualmente, el lavado de las manos permitió reducir entre $27 \%$ y $35 \%$ la morbilidad por EDA, mientras que la modificación de otras conductas de riesgo ayudó a disminuir el número de casos en $26 \%(2,11)$. El conjunto de estos factores, unido a una ablactación adecuada, contribuyeron a reducir la mortalidad por EDA entre 2 y $12 \%$. También se encontró que el uso de tazas y cucharas en lugar de biberones redujo las posibilidades de contaminación de los alimentos (2).

Según los resultados del análisis de cinco estudios controlados, la vitamina A puede contribuir a reducir la mortalidad por diarrea en niños pequeños. Llama la atención la uniformidad de estos hallazgos, a pesar de la diversidad de metodologías empleadas y las diferencias entre los países en los que se llevaron a cabo esos ensayos. Según sus resultados, con esta medida se logró disminuir la mortalidad entre 19 y $54 \%$, con riesgos relativos promedios de 0,71 (intervalo de confianza de $95 \%$ [IC95\%]: 0,57 a 0,88) (2). Sin embargo, los autores de este análisis consideran que la información disponible no es suficiente para confirmar esta hipótesis.

Algunos estudios han demostrado que los niños con bajo peso al nacer tienen el doble del riesgo de morir por diarrea que los niños que nacen con el peso adecuado para la edad gestacional (razón de posibilidades [odds ratio, $\mathrm{RP}]=2$; IC95\%: 1,1 a 4,4$)$ y que presentan mayor morbilidad por esta enfermedad, especialmente durante el primer año de vida $(8,9)$.

No se encontraron estudios que relacionen la oportunidad de la atención sanitaria con la gravedad de la EDA en Colombia. Estudios realizados en otros países indican que la mortalidad no es un buen indicador del impacto a corto plazo de los programas de salud, como sí lo son la morbilidad y la discapacidad (12).

Taylor, Emery y Carpenter (13) encontraron que una adecuada atención primaria permitió disminuir en $28 \%$ la mortalidad por diarrea en niños de 6 a 35 meses de edad y alcanzar una reducción total de $35 \%$ en la mortalidad en niños de 1 a 4 años. Esto podría indicar que el acceso oportuno al tratamiento adecuado puede ser esencial para reducir la mortalidad infantil (13-15).

En los últimos años, la atención se ha centrado en las causas de la diarrea, particularmente las de origen infec- cioso - causadas por parásitos, bacterias (principalmente Escherichia coli, Vibrio cholerae y especies de Salmonella y Shigella) o virus - por las posibilidades de tomar medidas preventivas $(3,6)$.

De los patógenos virales, el rotavirus ha recibido atención especial por su asociación con la deshidratación grave. Se calcula que la infección por rotavirus provoca al año 111 millones de episodios de gastroenteritis, 25 millones de consultas médicas, 2 millones de hospitalizaciones y entre 352000 y 592000 muertes, de las cuales $82 \%$ ocurren en los países más pobres (16). Este virus afecta principalmente a los niños menores de 3 años y alcanza valores máximos de incidencia en niños de 6 a 24 meses de edad. La mayoría de las infecciones son producidas por rotavirus de los serotipos $\mathrm{P} y$ $\mathrm{G}$, del grupo A (6).

El estudio de la epidemiología de la infección por rotavirus y del papel que este desempeña en la diarrea grave es de la mayor importancia para estimar la efectividad en función del costo de introducir en los esquemas nacionales de vacunación las vacunas que se encuentran actualmente en desarrollo. En Colombia, la epidemiología de este virus no está lo suficientemente bien caracterizada.

El objetivo del presente estudio fue establecer la relación entre la infección por rotavirus y la deshidratación por diarrea en niños colombianos menores de 5 años y determinar los factores de riesgo asociados con la diarrea con deshidratación ${ }^{3}$.

\section{MATERIALES Y MÉTODOS}

Se realizó un estudio de casos y testigos en la Clínica Infantil Colsubsidio, una institución de atención terciaria en Bogotá, Colombia, que cuenta con una unidad de hidratación que sigue los lineamientos promovidos por la Orga-

\footnotetext{
3 Un resumen de los resultados de este estudio fueron presentados en la Reunión Regional sobre la Implementación de la Vigilancia Epidemiológica de Rotavirus, organizada por la Organización Panamericana de la Salud en Lima, Perú, en septiembre de 2003 (17).
} 
nización Panamericana de la Salud (OPS) y la Organización Mundial de la Salud (OMS) $(18,19)$. Esta institución atendió en el año 2000 cerca de 3200 niños con EDA, 57\% de ellos menores de 5 años. De los pacientes atendidos en consultas por diarrea, $11 \%$ (352) requirieron hospitalización por complicaciones relacionadas con esa enfermedad y 4,5\% tuvieron complicaciones muy graves que hicieron necesaria su atención en la unidad de cuidados intensivos (20).

El tamaño de la muestra se calculó según la metodología descrita (21) para detectar un riesgo $\geq 2$, con un nivel de confianza de 0,05, una frecuencia esperada en el grupo testigo para cualquier factor de riesgo de 0,20 y un poder de 0,8 . La relación entre casos y testigos fue de 1:1. Se estimó la pérdida de información en 15\%; este valor se adicionó a la muestra calculada. El tamaño de muestra empleado fue de 290 niños.

El médico coordinador estableció las perdidas, a partir de las cuales se estimó y corrigió el movimiento del sesgo. La población se caracterizó al final de estudio y las pérdidas se corroboraron con los datos del registro obligatorio de atención de pacientes llevado por el departamento de estadísticas del hospital.

El grupo de casos estuvo compuesto inicialmente por 138 niños de 2 meses a 4 años de edad, hospitalizados con diagnóstico de EDA con deshidratación entre abril de 2000 y febrero de 2001. El grupo de testigos se conformó con 152 niños de la misma edad, que fueron atendidos por EDA sin deshidratación en la misma institución dentro de los 15 días siguientes a la captación del caso. El diagnóstico de EDA se estableció cuando el paciente presentaba al menos tres deposiciones líquidas diarias durante menos de 14 días. Los testigos que evolucionaron a casos fueron adicionados a este último grupo para el análisis.

Con el fin de reducir el sesgo de selección, se incluyeron en la muestra todos los casos que cumplieron con los criterios establecidos y los testigos fueron seleccionados aleatoriamente en las consultas ambulatorias diarias durante los horarios de la mañana y de la tarde (21).

Los testigos fueron citados a un control médico el primer y el quinto días después de su ingreso al estudio, con el fin de verificar la mejoría clínica, comprobar que no presentaron deshidratación durante la enfermedad y evaluar el tratamiento empleado. Estas visitas de control se realizaron en intervalos menores si los niños presentaban algún signo o complicación que lo justificara, o a solicitud de la madre. Cuando algún niño no asistió al control médico, se obtuvo la información de su estado de salud por vía telefónica. Cuando no fue posible establecer el estado final del cuadro diarreico de algún menor del grupo de testigos, el movimiento del sesgo se estimó de manera individual mediante la aplicación del modelo de regresión logística final con la información recolectada en el cuestionario aplicado el día en que el niño fue captado para el estudio.

Se excluyeron los pacientes que presentaban cualquiera de los siguientes: alguna enfermedad infecciosa concomitante; enfermedad diarreica por más de 14 días; intoxicación alimentaria; diagnóstico de enfermedad diarreica crónica o recurrente; antecedentes de alergia alimentaria; alguna enfermedad sistémica que favoreciera o cuya manifestación cardinal fuera la enfermedad diarreica; íleo paralítico de más de 24 horas de evolución; o residencia fuera del casco urbano de Bogotá.

Los médicos encargados de la valoración inicial de los pacientes recibieron una capacitación previa acerca del diagnóstico de EDA y de la toma de parámetros clínicos, según guías clínicas colombianas (22) y recomendaciones internacionales (23). El médico encargado de seleccionar los casos y los testigos, así como de verificar los criterios de inclusión y exclusión, de aplicar el cuestionario y de medir las variables clínicas de seguimiento de la enfermedad recibió una capacitación previa sobre EDA, la toma de parámetros clínicos, y los parámetros y conceptos necesarios para aplicar la encuesta.
A todos los niños se les realizó un examen físico completo. El grado de deshidratación se estableció según las recomendaciones de la OPS y la OMS (23). Las muestras de heces se tomaron en las primeras 48 horas después del ingreso del niño al estudio y se conservaron refrigeradas hasta su envío al laboratorio de virología del Instituto Nacional de Salud (INS) dentro de las siguientes 24 horas, donde se almacenaron a $-20{ }^{\circ} \mathrm{C}$ hasta su procesamiento. La presencia de rotavirus del grupo A se determinó mediante una prueba ELISA (Premier Rotaclone ${ }^{\circledR}$, Meridian Diagnostics, Inc., EUA). El control de la calidad de los resultados de laboratorio se realizó de acuerdo con los estándares internacionales (19).

Las madres de los menores respondieron un cuestionario elaborado especialmente para este estudio, con variables comparables para los casos y los testigos $(21,24)$. Además de las variables demográficas, socioeconómicas y ambientales, en la encuesta se recabó información acerca de la escolaridad, la edad, el estado civil y la actividad laboral de la madre o del cuidador del niño, de sus conocimientos, actitudes y hábitos de higiene personal y de sus posibilidades de acceder a los servicios de salud. Esta información se completó con datos sobre el estado nutricional del niño, su evolución clínica y los resultados de las pruebas de detección viral en las heces.

Para el análisis de los datos se emplearon los paquetes estadísticos Epi Info 6.04 (25) y Stata 5.0 (26). Se determinaron la frecuencia, la tendencia central y la dispersión de las variables continuas, así como las RP y sus intervalos de confianza de $95 \%$. El análisis bifactorial se realizó mediante la prueba de la ji al cuadrado o la prueba exacta de Fisher, según correspondiera. Se calcularon los valores de la ji al cuadrado para las tendencias y se controlaron los factores de confusión mediante la estratificación de las variables. Se escogió un nivel de significación de 0,05.

Las variables se introdujeron en un modelo logístico siguiendo un diseño jerárquico $(27,28)$ si los grupos deter- 
minados previamente tenían un valor de $P<0,05$ y el logaritmo de la razón de verosimilitudes era estadísticamente significativo $(P<0,05)$. El modelo final se estableció con variables de cada uno de los grupos, ajustadas según la edad y el sexo. Se calcularon los coeficientes y sus desviaciones estándar y se evaluó la presencia de sinergismo y correlación entre las variables.

El estudio cumplió con los requisitos éticos establecidos por la resolución No. 008430 de 1993, dictada por el Ministerio de Salud de Colombia, y fue aprobado por el Comité de Ética de la institución donde se realizó la investigación.

\section{RESULTADOS}

Todas las madres consultadas aceptaron participar en el estudio y la pérdida de información acerca de cualquiera de las variables fue menor de $10 \%$. Durante los 11 meses de estudio se entrevistó a las madres de 138 casos y de 152 testigos. Siete $(4,8 \%)$ de los testigos evolucionaron a casos y se consideraron como tales en el análisis de los resultados, por lo que tanto el grupo de casos como el de testigos quedaron finalmente compuestos por 145 niños. De los 290 menores estudiados, $153(52,8 \%)$ eran del sexo masculino y $137(47,2 \%)$ del femenino. La edad media fue de 19 meses (de 2 a 56 meses); $73 \%$ de los menores estudiados tenían menos de 2 años de edad.

El riesgo de presentar deshidratación fue mayor cuando las condiciones socioeconómicas de la familia del niño eran desfavorables. Estas condiciones se evaluaron según las características físicas de la vivienda, el acceso a los servicios públicos y la relación entre el número de personas que vivían en el hogar y el número de dormitorios de la vivienda (hacinamiento) (cuadro 1).

También se encontró una asociación entre el riesgo de presentar deshidratación y un peso al nacer menor de $2600 \mathrm{~g}(\mathrm{RP}=7,79$; IC95\%: 3,47 a 18,01) y la lactancia materna por menos de 3 meses (RP = 3,17; IC95\%: 1,66 a 6,13). El riesgo de deshidratación aumentó a
CUADRO 1. Variables generales asociadas con la deshidratación por diarrea en niños menores de 5 años. Bogotá, Colombia, 2000-2001

\begin{tabular}{|c|c|c|c|c|}
\hline Variable & Casos & Testigos & $\begin{array}{c}\text { Razón de } \\
\text { posibilidades }\end{array}$ & IC95\% ${ }^{a}$ \\
\hline \multicolumn{5}{|l|}{ Condiciones de la vivienda } \\
\hline Piso de cemento o tierra & 32 & 17 & 2,13 & $1,07-4,28^{b}$ \\
\hline $\begin{array}{l}\text { Más de dos personas duermen } \\
\text { en cada dormitorio }\end{array}$ & 34 & 7 & 6,15 & $2,47-16,0^{b}$ \\
\hline \multicolumn{5}{|l|}{ Escolaridad materna } \\
\hline Menor de 6 años & 12 & 3 & 5,21 & $1,35-29,37^{c}$ \\
\hline Entre 6 y 8 años & 34 & 13 & 3,41 & 1,63-7,22 \\
\hline Mayor de 8 años & 99 & 129 & 1,0 & \\
\hline \multicolumn{5}{|l|}{ Peso al nacer ${ }^{d}$} \\
\hline$<2600 \mathrm{~g}$ & 48 & 9 & 7,79 & $3,47-18,01^{b}$ \\
\hline$\geq 2600 \mathrm{~g}$ & 89 & 130 & 1,0 & \\
\hline \multicolumn{5}{|l|}{ Duración de la lactancia materna } \\
\hline Menos de 4 meses & 45 & 18 & 3,57 & $1,81-7,09$ \\
\hline Entre 4 y 6 meses & 37 & 37 & 1,43 & $0,79-2,59^{c}$ \\
\hline Más de 6 meses & 63 & 90 & 1,0 & \\
\hline Destete antes de los 4 meses & 79 & 21 & 7,07 & $3,85-13,06^{b}$ \\
\hline
\end{tabular}

medida que la escolaridad materna fue menor $\left(\chi^{2}\right.$ para la tendencia $\left.<0,001\right)$ y cuando la duración de la lactancia materna fue más breve $\left(\chi^{2}\right.$ para la tendencia $<0,001$ ) (cuadro 1 ).

La evaluación de los hábitos higiénicos de la madre mostró que el riesgo de deshidratación fue significativo cuando no se cumplieron las normas básicas de higiene durante la alimentación y la atención del menor (cuadro 2).

Algunas variables relacionadas con la falta de conocimientos previos acerca de la enfermedad y con los servicios de salud - no asistir a las consultas de control de crecimiento y desarrollo, la falta de información previa acerca de la diarrea o de la deshi-
CUADRO 2. Hábitos higiénicos asociados con la deshidratación por diarrea en niños menores de 5 años. Bogotá, Colombia, 2000-2001

\begin{tabular}{|c|c|c|c|c|}
\hline Hábito & Casos & Testigos & $\begin{array}{c}\text { Razón de } \\
\text { posibilidades }\end{array}$ & IC95\% ${ }^{a}$ \\
\hline $\begin{array}{l}\text { No lavar los alimentos antes } \\
\text { de darlos al niño }\end{array}$ & 42 & 14 & 3,83 & $1,89-7,87$ \\
\hline $\begin{array}{l}\text { Utilizar biberón para dar alimentos } \\
\text { al niño }\end{array}$ & 83 & 66 & 1,69 & $1,02-2,79$ \\
\hline $\begin{array}{l}\text { Madres que no se lavan las manos } \\
\text { después de ir al baño }\end{array}$ & 73 & 25 & 4,87 & $2,73-8,72$ \\
\hline $\begin{array}{l}\text { Madres que no se lavan las manos } \\
\text { después de cambiar los pañales } \\
\text { Madres que no se lavan las manos }\end{array}$ & 80 & 33 & 19,12 & $8,09-46,97$ \\
\hline $\begin{array}{l}\text { antes de dar la comida al niño } \\
\text { Madres que no lavan las manos }\end{array}$ & 55 & 19 & 4,05 & $2,15-7,67$ \\
\hline del niño antes de comer & 132 & 102 & 4,28 & $2,08-8,94$ \\
\hline
\end{tabular}


CUADRO 3. Variables relacionadas con los servicios de salud asociadas con la deshidratación por diarrea en niños menores de 5 años. Bogotá, Colombia, 2000-2001

\begin{tabular}{|c|c|c|c|c|}
\hline Variable & Casos & Testigos & $\begin{array}{c}\text { Razón de } \\
\text { posibilidades }\end{array}$ & $\mathrm{IC} 95 \%{ }^{\mathrm{a}}$ \\
\hline \multicolumn{5}{|l|}{ Niños que no asistieron a } \\
\hline y desarrollo & 56 & 27 & 2,75 & $1,56-4,87$ \\
\hline \multicolumn{5}{|l|}{ Madres que no han recibido } \\
\hline información sobre la diarrea & 86 & 50 & 2,77 & $1,67-4,62$ \\
\hline \multicolumn{5}{|l|}{ Madres que no saben qué es } \\
\hline la deshidratación & 59 & 21 & 4,05 & $2,21-7,46$ \\
\hline \multicolumn{5}{|l|}{ Madres que no saben para qué } \\
\hline sirve el suero oral & 59 & 21 & 4,06 & $1,70-13,13$ \\
\hline \multicolumn{5}{|l|}{ Madres que no saben reconocer } \\
\hline los signos de deshidratación & 88 & 12 & 18,26 & $8,78-38,72$ \\
\hline \multicolumn{5}{|l|}{ Detección de rotavirus en las heces } \\
\hline del menor & 74 & 51 & 1,92 & $1,16-3,18$ \\
\hline
\end{tabular}

a Intervalo de confianza de $95 \%$.

dratación, el conocimiento sobre el uso del suero oral, entre otros- mostraron una fuerte asociación con la deshidratación por diarrea (cuadro 3).

Se demostró la presencia de rotavirus en 125 de las 290 muestras fecales analizadas (43\%), la mayoría de ellas de niños de 6 a 24 meses $(72,8 \%)$. No se encontraron diferencias significativas según el sexo $(52 \%$ en niños frente a $48 \%$ en niñas; $P>0,05)$. Se observó un mayor riesgo de presentar deshidratación cuando en las muestras se detectó la presencia de rotavirus (cuadro 3 ). De los siete testigos que pasaron al grupo de casos por presentar deshidratación, cinco $(71,4 \%)$ tenían rotavirus en las heces.

La proporción de niños con rotavirus en las heces fue mayor a medida que aumentó el tiempo de estancia hospitalaria, mientras que los niños con resultados negativos mostraron una tendencia inversa (figura 1). El riesgo de tener una estancia hospitalaria mayor de 15 días cuando la prueba de detección de rotavirus en las heces era positiva fue 2,2 veces mayor que cuando no se detectaron rotavirus $(P<0,05)$.

Según el modelo de regresión logística seleccionado para el estudio, los factores de riesgo asociados significativamente $(P<0,05)$ con la deshidratación fueron un peso al nacer inferior a $2600 \mathrm{~g}$, el hacinamiento (cuando más de dos personas dormían en cada dormitorio), la lactancia materna por menos de 3 meses, el hábito de guardar los alimentos para alimentar posteriormente al niño, el hábito de las madres y cuidadores de no lavarse las manos después de ir al baño, y la detección de rotavirus en las heces del niño (cuadro 4). Como factor protector se encontró la mayor escolaridad materna (cuadro 4). Si bien los resultados del modelo logístico jerárquico no permitieron confirmar estadísticamente el efecto protector que desempeña la asistencia de los niños a las consultas de control de crecimiento y desarrollo en los últimos meses $(P=0,606)$, los resultados de la prueba de la razón de verosimilitudes indicaron que esta variable estaba asociada con una mejor evolución, por lo que debía tomarse en cuenta en el análisis.

Por último, después de hacer ajustes en función de todos los factores antes mencionados, el riesgo de presentar

FIGURA 1. Tiempo de estancia hospitalaria en menores de 5 años hospitalizados por diarrea con deshidratación, según los resultados de la detección de rotavirus por ELISA en las heces del menor. Bogotá, Colombia, 2000-2001

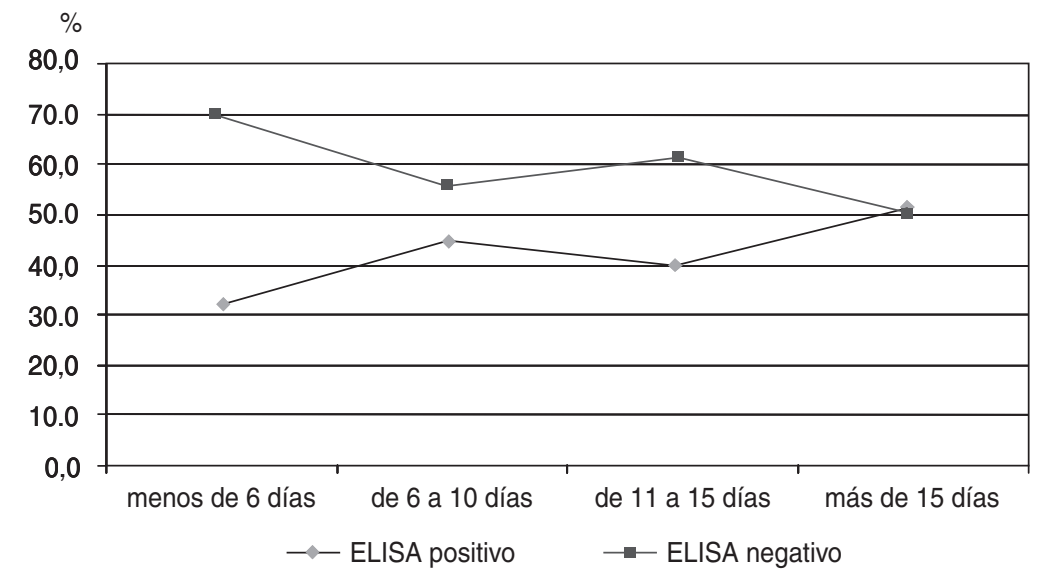


CUADRO 4. Variables asociadas con la deshidratación por diarrea en niños menores de 5 años de edad, según los resultados del modelo logístico jerárquico. Bogotá, Colombia, 2000-2001

\begin{tabular}{lccc}
\hline \multicolumn{1}{c}{ Variable } & Razón de posibilidades & IC95\% $\%^{\mathrm{a}}$ & $P^{\mathrm{b}}$ \\
\hline $\begin{array}{l}\text { Bajo peso al nacer }(<2600 \mathrm{~g}) \\
\begin{array}{l}\text { Más de dos personas duermen } \\
\quad \text { en cada dormitorio }\end{array}\end{array}$ & 3,22 & $1,32-7,84$ & 0,010 \\
$\begin{array}{l}\text { Escolaridad materna } \\
\text { Duración de la lactancia materna inferior }\end{array}$ & 2,41 & $1,04-5,59$ & 0,040 \\
$\quad$ a 3 meses & 0,83 & $0,73-0,95$ & 0,007 \\
$\begin{array}{l}\text { Guardar los alimentos algunas horas } \\
\quad \text { antes de alimentar al niño }\end{array}$ & 2,96 & $1,21-7,27$ & 0,017 \\
$\begin{array}{l}\text { Madres que no se lavan las manos } \\
\quad \text { después de ir al baño }\end{array}$ & 2,39 & $0,98-5,83$ & 0,053 \\
$\begin{array}{l}\text { Asistencia a consultas de control en } \\
\quad \text { los } 6 \text { meses anteriores a la encuestac }\end{array}$ & 4,28 & $1,90-9,64$ & $<0,001$ \\
$\begin{array}{l}\text { Detección de rotavirus en las heces } \\
\text { del menor }\end{array}$ & 0,95 & $0,78-1,15$ & 0,606 \\
\hline
\end{tabular}

a Intervalo de confianza de $95 \%$

${ }^{b}$ Nivel de significación $P<0,05$.

' La escolaridad materna y la asistencia a consultas de control de crecimiento y desarrollo se consideraron variables continuas en el modelo (de 0 a 19 años de estudio y de 0 a 6 controles, respectivamente)

deshidratación aumentó a 3,4 (IC95\%: $1,71$ a 7,00$)$ cuando se detectó rotavirus en las heces.

\section{DISCUSIÓN}

Los resultados del presente estudio demuestran que las complicaciones por EDA pueden reducirse mediante la puesta en marcha de estrategias adecuadas en la atención primaria, las cuales pueden ser muy efectivas en función del costo. Si bien se ha descrito que la mortalidad disminuye cuando la población tiene acceso adecuado a los servicios de salud (3, 12-15), en este estudio se demostró que la mala calidad de las acciones de promoción y prevención llevadas a cabo en esta población con fácil acceso a los servicios de salud determinó el aumento de la morbilidad, favoreció el desarrollo de complicaciones - algunas de ellas graves- e incrementó los costos de la atención sanitaria.

No se determinaron las causas por las que una población que cuenta con un sistema de seguridad social de salud carece de conocimientos básicos acerca de la prevención de enfermedades diarreicas. Esta situación es preo- cupante, ya que el Estado ha destinado una gran cantidad de recursos para garantizar que las acciones de promoción de salud y prevención de enfermedades lleguen a toda la población. Además, estas medidas no requieren de una elevada capacitación de los trabajadores sanitarios y son fáciles de poner en práctica. Los resultados expuestos resaltan la importancia de estas acciones y la necesidad de que los programas de salud pública sean evaluados sistemáticamente después de su puesta en marcha.

La mayor parte de los conceptos educativos que permitirían disminuir las complicaciones por diarrea en los países en desarrollo están contenidos en la estrategia Atención Integrada de las Enfermedades Prevalentes en la Infancia (AIEPI), promovida por la OPS y la OMS (23). Esta estrategia hace hincapié en la necesidad de generar conocimientos, prácticas y habilidades en las madres, los trabajadores comunitarios y el personal sanitario, con el fin de prevenir y detectar tempranamente las complicaciones y favorecer la consulta médica oportuna.

Debe tenerse en cuenta la necesidad de ajustar estas estrategias a los conceptos culturales, sociales y educativos de la población, de forma que se favorezcan el conocimiento, el bienestar y la salud de la comunidad. Igualmente, se debe estimular el desarrollo en la comunidad de una cultura de salud que sea entendida como un derecho - para garantizar la equidad-y como un deber. En esa cultura, la familia debe ser responsable, individual y colectivamente, de la salud de los niños.

Estos resultados también corroboran la estrecha relación existente entre el bajo peso al nacer y el aumento de la morbilidad por EDA $(8,9)$. Teniendo en cuenta el papel importante que desempeña este factor en la aparición de complicaciones por enfermedades infecciosas y no infecciosas, es necesario evaluar conjuntamente las estrategias de salud pública dirigidas a las madres y cuidadores de menores de 5 años y a las mujeres en edad fértil, de manera que las intervenciones resulten más eficaces y efectivas en función del costo.

La relación existente entre el nivel socioeconómico bajo, las medidas higiénicas y la morbilidad por diarrea encontrada en este estudio confirma los resultados de otros investigadores y resalta el impacto negativo del bajo nivel socioeconómico y de los hábitos higiénicos inadecuados de algunas madres y cuidadores en la salud de los niños $(2,3,10,11)$.

Uno de los hallazgos de mayor interés es la relación encontrada entre la morbilidad por EDA y la infección por rotavirus, observada también por otros autores $(1-3,6,19)$. Aunque no se investigó la presencia de infecciones simultáneas por rotavirus y otros virus y bacterias, es posible que este tipo de infección produzca una enfermedad de mayor gravedad.

Un elevado porcentaje de las muestras de heces resultó positivo en la prueba de detección de rotavirus (43\%), especialmente en niños de 6 a 24 meses de edad. Estudios anteriores realizados en Colombia encontraron valores similares a los hallados en esta investigación. En 1982, en Medellín se demostró la presencia de rotavirus en $48 \%$ de los pacientes hospitalizados con diarrea grave y en $36 \%$ de los que presentaban diarrea moderada (29). 
En otra investigación realizada en Cartagena se aisló rotavirus en $40,4 \%$ de las muestras fecales analizadas entre 1998 y 1999 (30).

A diferencia de los estudios anteriores, en Bogotá y Pereira se aisló rotavirus en solo $20 \%$ de las muestras fecales de niños y adultos con diagnóstico de EDA (31). Este bajo porcentaje puede deberse a que la infección por este virus transcurre frecuentemente de forma asintomática después de los 36 meses de edad.

Para evaluar el beneficio de introducir una vacuna contra la infección por rotavirus en el esquema nacional de vacunación, una vez que se encuentre disponible, y calcular la efectividad de esta medida en función del costo, se debe determinar la estacionalidad del rotavirus, definir su relación con la gravedad de la diarrea e identificar los serotipos circulantes más frecuentes. Para ello se requiere establecer sistemas nacionales de vigilancia que se articulen entre sí, de manera que se logren las ventajas de la economía de escala al introducir la vacuna en los programas de salud pública de los países de la Región. Además, es necesario que los organismos internacionales vinculados con la salud infantil, junto con los gobiernos, estudien las vías de financiar la introducción de nuevos productos biológicos en los programas nacionales de vacunación.

Aunque el mecanismo protector de la lactancia materna frente al rotavirus no se ha dilucidado por completo, se ha demostrado el efecto positivo que tiene esta práctica en la madre y el niño. Fomentar la lactancia materna contribuiría al desarrollo integral del niño y a reducir la morbilidad y la mortalidad por diarrea y por otras enfermedades $(8,9,23,32-41)$.

La asociación observada entre la presencia de rotavirus y el tiempo de estancia hospitalaria puede ser explicada total o parcialmente por la presencia de infecciones mixtas por rotavirus y algunas bacterias, especialmente Escherichia coli, Salmonella spp. y
Shigella spp. La coinfección puede originarse en la comunidad o puede estar favorecida por la deshidratación grave o el choque hipovolémico de los niños enfermos. Sin embargo, teniendo en cuenta el alto porcentaje de niños que estuvieron hospitalizados durante tres días o más, la coinfección pudo haberse adquirido en el hospital. Para aclarar esta posible relación causal se requieren estudios adicionales.

Aunque los parámetros clínicos que definen la deshidratación se han estudiado ampliamente y se ha comprobado su sensibilidad y especificidad, en esta investigación se cotejaron las notas del médico que ingresó a cada paciente con las generadas por los médicos de sala y las auxiliares de enfermería; además, se revisó el balance de líquidos de cada niño. Para compensar el sesgo de referencia, los niños y niñas del grupo de testigos se seleccionaron en el servicio de consulta externa de la misma institución donde se captaron los casos. No obstante, es posible que se haya encontrado mejor nivel económico en las familias de los niños del grupo de testigos, ya que esta puede ser una población cautiva que asiste a las consultas de la institución donde se realizó el estudio, pero que se hospitaliza en otras instituciones. El sesgo de memoria se compensó por el tiempo corto que medió entre la exposición y la recolección de la información - tanto para los casos como para los testigos-, por el hecho de que los niños de ambos grupos tenían la enfermedad en el momento de la entrevista y porque fueron las madres en persona la fuente principal de información. Cuando esto último no fue posible, se entrevistó telefónicamente a la madre.

Adicionalmente, el número de entrevistadores se redujo al mínimo posible, el cuestionario fue validado con antelación y la información se revisó periódicamente para detectar posibles sesgos. El bloque socioeconómico se midió a partir de preguntas del censo nacional y de estudios que han demos- trado una concordancia superior a $80 \%$ entre las características físicas de la vivienda y el estado socioeconómico de la familia $(8,9,27,28)$.

Por último, a pesar de que no se tipificaron los aislamientos virales para determinar los serotipos de rotavirus circulantes, las muestras se mantienen conservadas a $-20{ }^{\circ} \mathrm{C}$ para realizar este análisis una vez que se cuente con los recursos necesarios.

Los resultados de este estudio muestran que la calidad inadecuada de las acciones de promoción y prevención en una población con fácil acceso a los servicios de salud favoreció la aparición de casos de diarrea con deshidratación, con el consiguiente aumento de los costos de la atención sanitaria. Igualmente, demuestran que el rotavirus desempeña un papel importante en la deshidratación por EDA. Es necesario establecer un sistema de vigilancia que se articule con los de otros países de la Región, con el fin de evaluar la efectividad en función del costo de la vacunación contra el rotavirus una vez que la vacuna se encuentre en el mercado.

Para disminuir la morbilidad por diarrea se requieren el compromiso y las acciones conjuntas de las autoridades, la comunidad y todos los actores que de una forma u otra están vinculados con la salud y el bienestar de los niños; la toma de decisiones y la elaboración de políticas basadas en los resultados científicos; y la evaluación sistemática de los programas de promoción y prevención. En estas condiciones, los recursos tecnológicos disponibles o en desarrollo - como las vacunas- serían más eficientes y eficaces y tendrían un mayor impacto en la salud pública.

Agradecimientos. Los autores agradecen a Elmer Escobar y Amparo Buendía la revisión crítica de este documento y sus invaluables aportes, y al personal de la Clínica Colsubsidio, sin el cual no hubiera sido posible culminar con éxito este estudio. 


\section{REFERENCIAS}

1. Bern C, Martínez J, de Zoysa I, Glass R. The magnitude of the global problem of diarrhoeal disease: a ten-year update. Bull World Health Organ. 1992;70(6):705-14.

2. Huttly SRA, Morris SS, Pisani V. Prevention of diarrhoea in young children in developing countries. Bull Word Health Organ. 1997; 75(2):163-74.

3. Cunliffe NA, Kilgore PE, Bresee JS, Steele AD, Luo N, Hart CA, et al. Epidemiology of rotavirus diarrhoea in Africa: a review to assess the need for rotavirus immunization. Bull World Health Organ. 1998;76(5):525-37.

4. Colombia, Ministerio de Salud. La carga de la enfermedad en Colombia. Santa Fe de Bogotá: Ministerio de Salud de Colombia; 1994.

5. Colombia, Ministerio de Salud. SIS 12. Base de datos de la Oficina de Sistemas de Información. Santa Fe de Bogotá: Ministerio de Salud; 1989-1997.

6. Offit PA, Clark HF. Rotavirus. Capítulo 139. En: Mandell GL, Bennett JE, Dolin R, eds. Mandell, Douglas and Bennett's principles and practice of infectious diseases. 5 . $^{\mathrm{a}} \mathrm{ed}$. New York: Churchill Livingstone; 2000. Pp. 16961703.

7. Nizami SQ, Bhutta ZA, Molla M. Efficacy of traditional rice-lentil-yogurt diet, lactose-free milk, protein-based formula, and soy protein formula in management of secondary lactose intolerance with acute childhood diarrhoea. J Trop Pediatr. 1996;42:133-7.

8. Victora GC, Smith PG, Vaughan JP, Nobre LC, Lombardi C, Texeira AM, et al. Infant feeding and deaths due to diarrhea: a case-control study. Am J Epidemiol. 1989;129:1032-41.

9. Victora GC, Smith PG, Vaughan JP. Evidence for protection by breast-feeding against infants deaths from infectious diseases in Brazil. Lancet. 1987;21:319-22.

10. Ahmed F, Clemens JD, Rao MR, Sack DA, Khan MR, Haque E. Community based evaluation of the effect of breast-feeding on the risk of microbiologically confirmed or clinically presumptive shigellosis in Bangladeshi children. Pediatrics. 1992;90:406-11.

11. Peterson AE, Roberts L, Toole MJ, Peterson DE. The effect of soap distribution on diarrhoea: Nyamithuthu Refugee Camp. Int J Epidemiol. 1998;27:520-4.

12. Chen L, Hill A, Murray CJL. A critical analysis of design, results and implications of the mortality and use of health services surveys. Int J Epidemiol. 1993;22(Supl1):73-80.

13. Taylor EM, Emery JL, Carpenter RG. Identification of children at risk of unexpected death. Lancet. 1983;2(8357):1033-4.

14. Taylor WR, Chahnazarian A, Weinman J, Wernette M, Roy J, Pebley AR, et al. Mortality and use of health services surveys in rural Zaire. Int J Epidemiol. 1993;22(Supl1):15-9.

15. Chahnazarian A, Ewbank D, Makani B, Ekouevi K. Impact of selective primary care on childhood mortality in rural health zone of Zaire. Int J Epidemiol. 1993;22(Supl1):32-41.
16. Parashar D, Hummelman EG, Bresse JS, Miller M, Glass R. Global illness and deaths caused by rotavirus disease in children. Emerging Infect Dis. 2003;9(5):565-72.

17. Organización Panamericana de la Salud. Generando información para la toma de decisiones. Informe final de la Reunión regional sobre la implementación de la vigilancia epidemiológica de rotavirus, Lima, Perú, septiembre de 2003. Washington, D.C.: OPS; 2003. (PAHO/FCH/IM/2003-00005).

18. World Health Organization. Guidelines for research on acute respiratory infections: memorandum from a WHO meeting. Bull World Health Organ. 1982;60:521-33.

19. World Health Organization. Generic protocols for (i) hospital-based surveillance to estimate the burden of Rotavirus gastroenteritis in children and (ii) a community-based survey on utilization of health care services for gastroenteritis in children. Field test version. Geneva: WHO; 2002.

20. Colombia, Clínica Colsubsidio. Registro de pacientes. Reporte epidemiológico. Santa Fe de Bogotá: Clínica Colsubsidio; 2000.

21. Schlesselman JJ. Case-control studies design, conduct, analysis. London: Oxford University Press; 1982.

22. Colombia, Clínica Colsubsidio. Guías para el manejo de la enfermedad diarreica aguda. Santa Fe de Bogotá: Clínica Colsubsidio; 2000.

23. Colombia, Ministerio de Salud, Organización Panamericana de la Salud. Atención integrada a las enfermedades prevalentes de la infancia. Santa Fe de Bogotá: Ministerio de Salud, OPS; 2000.

24. Bennett AE, Ritchie K. Questionnaires in medicine. A guide to their design and use. London: Oxford University Press; 1975.

25. Centers for Disease Control and Prevention, World Health Organization. Epi-Info 6.04d Atlanta: CDC, WHO; 2001

26. Stata Corporation. Stata 5.0, statistics/data analysis. College Station, Texas: Stata Corporation; 1984-1997.

27. Victora CG, Huttly SR, Costa SC, Olinto MT. The role of conceptual frameworks in epidemiological analysis: a hierarchical approach. Int J Epidemiol. 1997;26:224-7.

28. Cáceres DC, Alzate RA. Factores de riesgo para ser hospitalizado por neumonía en niños menores de 5 años en el Hospital Infantil Club Noel de Cali, 1997. Premio Colsubsidio de Investigación en Pediatría. IV Bienal de Pediatría. Colsubsidio-Américas. Santa Fe de Bogotá: OP Gráficas; 1999.

29. Trujillo H, Jaramillo C, Restrepo M, Mejía GI, Zapata TC, Ramírez R, et al. Rotavirus y otros enteropatógenos en la etiología de la diarrea aguda en Medellín, Colombia, 1982. Bol Oficina Sanit Panam. 1985;98(3): 251-61.

30. Máttar $S$, Pulido $N$, Mulett $R$, Londono $D$ Medina G, Martínez M, et al. Aetiology of acute infectious diarrhoea in a private hospital in Colombia. Med Sci Res. 1999;27:29-32.
31. Bermeo L, Mogollón D, Ariza F, Barrera J, Jerabek L, Gutiérrez MF. Molecular characterization of rotavirus strains obtained from human diarrheic samples and their epidemiological implications. Universitas Scientiarum. 1997; 4(1):71-81.

32. Holberg CJ, Wright AL, Martinez FD, Ray CG, Taussig LM, Lebowitz MD. Risk factors for respiratory syncytial virus-associated lower respiratory illnesses in the first year of life. Am J Epidemiol. 1991;133:1135-51.

33. World Health Organization. Division of Diarrhoeal and Acute Respiratory Disease Control, Interim report, 1994. Geneva: WHO; 1995. (WHO/CDR/95)

34. Paxton LA, Reed SC, Steketee RW, Otieno JO, Nahlen B. An evaluation of clinical indicators for severe pediatric illness. Bull World Health Organ. 1996;74(6):613-8.

35. Graham NM, Woodward AJ, Ryan P, Douglas RM. Acute respiratory illness in Adelaide children. II: The relationship of maternal stress, social support and family functioning. Int J Epidemiol. 1990;19:937-44.

36. Suwanjutha S, Ruangkanchanasetr $S$, Chantarojanasiri $\mathrm{T}$, Hotrakitya S. Risk factors associated with morbidity and mortality of pneumonia in Thai children under 5 years. Southeast Asian J Trop Med Public Health. 1994;25(1):60-6.

37. Fuchs SC, Victora CG, Wannmacher L, Goncalves C, Barbosa A, Menta L, et al. Reliability of environmental, biological and health characteristics of infants from a developing country. Int J Epidemiol. 1996;25:1005-8.

38. Margolis PA, Greenberg RA, Keyes LL, LaVange LM, Chapman RS, Denny FW, et al. Lower respiratory illness in infants and low socioeconomic status. Am J Public Health. 1992;82:1119-26.

39. Snow RW, Chellenberg JR, Forster D, Mung'ala VO, Marsh K. Factors influencing admission to hospital during terminal childhood illness in Kenya. Int J Epidemiol. 1994; 23:1013-9.

40. Lombrail P, Vitoux-Brot C, Bourrillon A Brodin M, De Pouvourville G. Another look at emergency room overcrowding: accessibility of the health services and quality of care. Int J Qual Health Care. 1997;9:225-35.

41. Goodman DC, Fisher E, Stukel TA, Chang C. The distance to community medical care and the likelihood of hospitalization: is closer always better? Am J Public Health. 1997;87(7): 1144-50.

Manuscrito recibido el 16 de abril de 2004. Aceptado para publicación, tras revisión, el 14 de octubre de 2004. 
ABSTRACT Objectives. To establish the relationship between rotavirus infection and dehydration from diarrhea in Colombian children under 5 years of age, and to identify risk factors for diarrhea with dehydration. Acute diarrheal disease:
a public health challenge
in Colombia

Key words
Methods. A case-control study was performed in an urban hospital in Bogotá, Colombia, between April 2000 and February 2001. The sample was composed of 290 children of both sexes under 5 years of age; of these children, 145 of them were hospitalized for acute diarrheal disease (ADD) with dehydration (cases), and 145 had a diagnosis of ADD but no signs of dehydration (controls). All children underwent a complete physical examination. Mothers responded to a questionnaire containing items on demographic and socioeconomic variables, as well as on knowledge, attitudes, and practices with regard to hygiene, and on access to health services.

Results. An association was detected between diarrhea with dehydration and the presence of rotavirus in fecal samples (odds ratio [OR] $=3.46 ; 95 \%$ confidence interval [95\% CI]: 1.71 to 7.00$)$, birth weight $<2600 \mathrm{~g}(\mathrm{OR}=7.79 ; 95 \% \mathrm{CI}: 3.47$ to 18.01$)$, and breastfeeding for less than 3 months $(\mathrm{OR}=3.17$; 95\% CI: 1.66 to 6.13$)$. The risk of having dehydration was associated with low socioeconomic status, poor hygienic practices among the child's family members, and mother's low educational level.

Conclusions. The ineffectiveness of health promotion and disease prevention activities in a population with easy access to health services set the stage for the appearance of cases of diarrhea with dehydration. Rotavirus infection plays an important role in the severity of ADD among Colombian children.

Diarrhea, infantile; dehydration; risk factors; rotavirus; Colombia.

It may be possible to incorporate laughter into daily activities, just as is done with other heart-healthy activities, such as taking the stairs instead of the elevator. The recommendation for a healthy heart may one day be exercise, eat right and laugh a few times a day.

[Es factible que la risa se incorpore a las actividades cotidianas, al igual que cualquier otra actividad que es buena para el corazón, como usar las escaleras en lugar del ascensor. Puede que algún día los cardiólogos recomienden hacer ejercicio, comer sanamente y reír varias veces al día.]

Michael Miller

Center for Preventive Cardiology, University of Maryland Medical Center / Centro de Cardiología Preventiva, Universidad de Maryland 